NSF-ITP-97-141

hep-th/9711097

\title{
The Shape of Branes Pulled by Strings
}

\author{
Akikazu Hashimoto \\ Institute for Theoretical Physics \\ University of California \\ Santa Barbara, CA 93106-4030
}

\begin{abstract}
We examine the system where a string stretches between pair of D-branes, and study the bending of the D-brane caused by the tension of the string. If the distance between the pair of D-branes is sent to infinity, the tension of the string stretching between them is strong enough to pull the spike all the way to infinity. We study the shape of these spikes when the branes are finite distance apart using two different methods. First, we consider a string stretched between a pair of D2-branes in type IIA theory by going to the M-theory limit in which all of these branes are M-theory 2-branes embedded along a holomorphic curve. Second, we consider a D-string stretched between a pair of D3-branes in type IIB theory and infer the geometry of the D3-brane embeddings from the configuration of the adjoint scalar field in the magnetic monopole solution of Prasad and Sommerfield. The case of fundamental string stretching between a pair of D3-branes follows from S-duality. The energy of these configurations matches the expected value based on fundamental string and D-string tensions.
\end{abstract}

November 1997 


\section{Introduction}

In the recent years, brane objects have emerged in the forefront as the key ingredient in the study of string theory and field theories. Branes are extended objects in space-time; when they occupy $p$-dimensional volume in space, they are referred to as $p$-branes. Fundamental particles and strings are also branes, in a sense that they could be thought of as 0-branes and 1-branes. Other types of branes can appear as a soliton of the theory, and can be classified according to its dimension and its charge. Some of these branes admits a simple perturbative description as D-branes [1].

These brane objects have found spectacular range of applications. One active avenue of investigation in the recent years have been the brane construction of quantum field theories. The basic idea stems from the fact that the world volume of D-branes contains a U(1) gauge field in its massless open string spectrum. When $N$ parallel D-branes approach each other, the $U(1)^{N}$ gauge symmetry is enhanced to $U(N)$ since the "W-bosons" corresponding to strings stretching between the branes also become massless [2]. Various matter content can be added to the theory, for example by including orthogonally intersecting branes [3, 4, 5].

A configuration of interest from the point of view of the brane construction of gauge theories is the one where an open $p$-brane ends on another brane [6]. One can describe, for example, a magnetic monopole in $3+1$ dimensional $S U(2)$ gauge theory in terms of a pair of parallel D3-brane with an orthogonal D1-brane ending on the D3-branes 《7, 8]. Other configuration of branes stretching between branes can also be dynamically generated when certain class of orthogonal D-branes cross as they move past one another in space [4, 5, 9, 10, 11].

The branes which appear in string theory generally have tensions of order $1 / g$ or $1 / g^{2}$ where $g$ is the string coupling constant. Therefore, for arbitrarily small but finite $g$, these branes have finite tension and will bend slightly when some other brane is attached and exerts its tension. This effect was studied systematically for the system consisting of a pair of Neveu-Schwarz five-branes in type IIA theory, suspending a collection of open D4-branes with endpoints along the Neveu-Schwartz five brane world volume [12]. The bending of the NS5-brane far away from the D4-brane was given a natural interpretation as running of the coupling constant for the D4-brane world volume theory. The geometry of NS5-brane near the D4-brane was studied by taking advantage of M-theory limit in which both the NS5brane and the D4-brane becomes an M-theory five brane. BPS configurations in this limit corresponds to holomorphic embeddings of the world volume into space time [13]. Such an embedding is generically smooth, but by shrinking the radius of the 11-th dimension, this smooth configuration degenerates into a singular geometry of D4-branes with endpoints on Neveu-Schwarz 5-branes. (A closely related configuration of D2-brane stretching between NS5-branes and D4-branes was discussed in [14.)

In this article, we attempt to address the question "what is the shape of D-branes which are pulled by strings." In other words, we will consider a system where an open string has 
one of its endpoints on a D-brane円, and study the bending of the D-brane in response to the tension exerted by the string. We will work in the context of weakly coupled string theory, where the brane geometry is expected to contain singularities. Understanding the nature of this singularity will be one of the goals of this paper. To be concrete, we will focus on the geometry of the bending of D3-branes due the tension of either a fundamental string or a D-string which ends on the worldvolume of the D3-brane [7, 8].

An important step in addressing this question was made in recent papers [17, 18]. (See 19, 20, 21 for related work.) These authors considered an electric BPS field configurations of the Dirac-Born-Infeld (DBI) action ( $p=3$ for D3-brane)

$$
S=T_{p} \int d^{p+1} x \sqrt{-\operatorname{det}\left(\eta_{\mu \nu}+T^{-1} F_{\mu \nu}+\partial_{\mu} X^{i} \partial_{\nu} X_{i}\right)}
$$

These electric charges correspond to endpoints of fundamental strings. The BPS configuration they found was of the form (in Coulomb gauge)

$$
T^{-1} A_{0}=c_{p} / r^{(p-2)}, \quad X^{9}=c_{p} / r^{(p-2)} .
$$

Here, $p=3, T=\left(2 \pi \alpha^{\prime}\right)^{-1}$, and

$$
T_{p}=\frac{1}{g}(2 \pi)^{(1-p) / 2} T^{(p+1) / 2}, \quad c_{p}=\frac{g}{(p-2) \Omega_{p-1}}(2 \pi)^{(p-1) / 2} T^{-(p-1) / 2} .
$$

The scalar field $X_{9}$ encodes the embedding of the D3-brane into space-time which contains an infinite spike at $r=0$ extending in the $x_{9}$ direction. The energy density for this configuration is

$$
T_{00}(r)=\frac{c_{p}^{2}(p-2)^{2} T_{p}}{r^{2(p-1)}}
$$

The total energy of this configuration is the integral of this expression over the 3-brane world volume

$$
E=\int d^{p} r \frac{c_{p}^{2}(p-2)^{2} T_{p}}{r^{2(p-1)}}=c_{p}(p-2) \Omega_{p-1} T_{p} \int d r \frac{(p-2) c_{p}}{r^{p-1}} .
$$

Although this integral is formally diverging near $r=0$, its physical origin can be elucidated by making a change of variables

$$
d X_{9}=d r \frac{(p-2) c_{p}}{r^{p-1}}
$$

Also noting that $c_{p}(p-2) \Omega_{p-1} T_{p}=T$, the expression for the energy of the system becomes

$$
E=T \int d X_{9}
$$

This expression has natural interpretation as the energy of string with tension $T$ stretching in the $X_{9}$ direction. This lead the authors of [17, 18] to interpret the semi-infinite spike on the 3-brane world volume as the string itself.

\footnotetext{
${ }^{1}$ The same system in the perturbative context was also studied in [15]. Similar situation arises also in the context of topological defect solutions in ordinary field theories [16].
} 


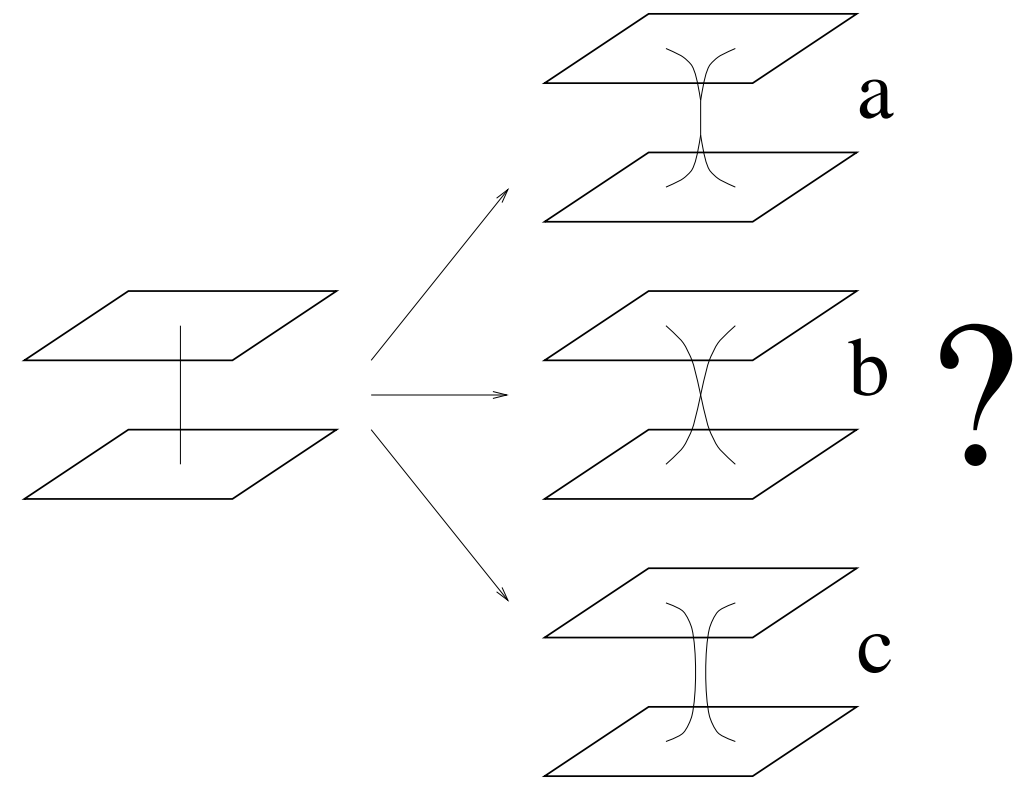

Figure 1: A string stretches between pair of parallel D3-branes, causing the D3-branes to bend.

This picture runs into problems, however, if one wishes to consider a configuration where a string stretches between a pair of parallel 3-branes separated at a finite distance, say at $X_{9}=-b$ and $X_{9}=b$, respectively. One might imagine that near $X_{9}=-b$ or $X_{9}=b$, the brane configuration is given by the BPS configuration described above, i.e. $X_{9}=-b+c_{3} / r$ and $X_{9}^{\prime}=b-c_{3} / r$. The difficulty arisis at the point in $r$ where $X$ and $X^{\prime}$ meets, since these curves do not interpolate naturally into each other. Natural expectation is that in the presense of the second D3-brane, the geometry of the bending of the D3-branes is modified near $r=0$. There are three qualitative possibility for how this might come about. One possiblity is for the D3-brane to be pinched off and collapse to zero radius at finite distance before it meets the other D3-brane (figure 1.a). One would expect a string to stretch between the points where the D3-branes are pinched off. Another possiblity is for the two D3-branes to meet precicely at the point where the radius shrinks to zero (figure 1.b). Yet another possiblity is for the pair of D3-branes to smoothly interpolate each other in a wormhole type geometry (figure 1.c).

Possibility 11.c has been discussed by authors of [17, 18]. They considered a cantenoidal static configuration of the Born-Infeld action of the form

$$
\begin{aligned}
E_{r} & =\frac{A}{\sqrt{r^{2 p-2}-r_{0}^{2 p-2}}} \\
\partial_{r} X_{9} & =\frac{B}{\sqrt{r^{2 p-2}-r_{0}^{2 p-2}}}
\end{aligned}
$$

where the constant $r_{0}^{2 p-2}=B^{2}-A^{2}$ controls the size of the throat, and $A=(p-2) c_{p}$ is 
fixed by the charge of the string endpoint. Unfortunately, such a configuration can only describe a 3-brane anti-3-brane configuration, as the orientation of the brane reverses as one interpolates down the throat.

In the remainder of this paper, we will discuss different approaches for investigating the geometry of the brane configurations illustrated in figure 1. Note that since the configuration in figure 1 contains a set of parallel D3-branes, the full non-abelian generalization of the Born-Infeld action is required in order to study this system along the lines of [17, 18]. Unfortunately, no satisfactory formulation of such an action is currently available. (Tseytlin has recently proposed a concrete formulation of the non-abelian Born-Infeld action using the symmetrized trace [22. Although this proposal passes many tests, some issues remain in understanding its fluctuation spectrum around a non-trivial background [23].) We will therefore approach this problem using indirect methods. We begin by considering more general class of static solutions for Born-Infeld action which appears to describe spikes being pinched off to a point and degenerating to a string as illustrated in figure 1.a. This approach turns out to be problematic from the point of view of energy. Two alternative methods for studying the shape of the bending is discussed. First, we consider a closely related system of a string stretching between D2-branes in type IIA theory, where one can exploit known facts from M-theory by extrapolating the coupling constant to strong coupling. Unfortunately, it is not clear how this approach can be extended to the case of D3-branes. The second approach is to consider the Yang-Mills approximation to the Born-Infeld action. In this approach, the shape of the branes is read off from the field configuration of the Higgs scalar in the Prasad-Sommerfield magnetic monopole solution 24] for the SU(2) gauge theory. Energies for these configurations can be computed and gives the expected results. In the abelian case, the BPS field configuration for the Maxwell theory turned out to also satisfy the BPS condition in the Born-Infeld theory [17, 18]. Perhaps for BPS configurations, the Yang-Mills and Born-Infeld action is supposed to give same answers even for non-abelian gauge theories. If true, this idea may provide a useful guiding principle for the formulation of non-abelian Born-Infeld actions. (We will show in Appendix A that this property holds for Tseytlin's action [22.) The fact that the energies of these configurations work out exactly provides some support for this idea.

\section{Static solutions of Dirac-Born-Infeld action}

Let us begin by looking for a static configuration of the Dirac-Born-Infeld action which might capture the geometry of the brane configuration of figure 1. For concreteness, let us concentrate on 3+1 dimensional Born-Infeld theory with 6 scalars. For our purpose here, we can set all fields other than the gauge potential $\phi=T^{-1} A_{0}$ and one of the transverse scalar $y=X_{9}$ (say) to zero. Since we are interested in static configurations, we can also set all

\footnotetext{
${ }^{2}$ I am grateful to Juan Maldacena for pointing out this approach to me.
} 


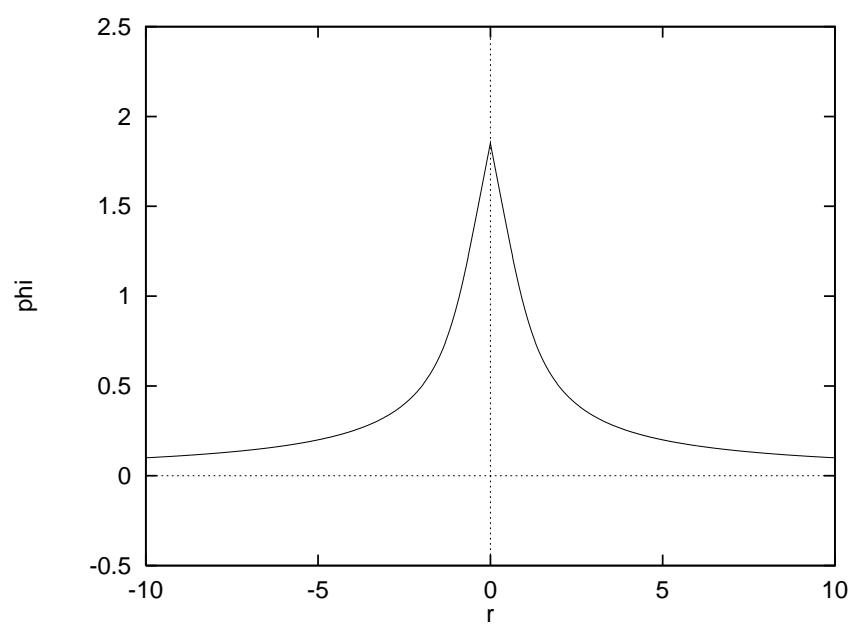

Figure 2: Electrostatic potential (in units of $\left[(p-2) c_{p}\right]^{\frac{1}{p-1}}$ ) for a BIon solution in $3+1$ dimensional Born-Infeld theory.

time-derivatives to zero. The statics problem then reduces to extremization of [18]

$$
T_{p} \int d^{p} x \sqrt{1+|\nabla y|^{2}-|\nabla \phi|^{2}+(\nabla y \cdot \nabla \phi)^{2}-|\nabla \phi|^{2}|\nabla y|^{2}}
$$

which gives rise to equations of motion

$$
\begin{aligned}
& \nabla \cdot \frac{-\nabla \phi+\nabla y(\nabla y \cdot \nabla \phi)-\nabla \phi(\nabla y)^{2}}{\sqrt{1-(\nabla \phi)^{2}+(\nabla y)^{2}+(\nabla y \cdot \nabla \phi)^{2}-(\nabla y)^{2}(\nabla \phi)^{2}}}=0 \\
& \nabla \cdot \frac{\nabla y+\nabla \phi(\nabla y \cdot \nabla \phi)-\nabla y(\nabla \phi)^{2}}{\sqrt{1-(\nabla \phi)^{2}+(\nabla y)^{2}+(\nabla y \cdot \nabla \phi)^{2}-(\nabla y)^{2}(\nabla \phi)^{2}}}=0 .
\end{aligned}
$$

The approach of this section is motivated by the existence of static charged field configuration [25] (referred to as a "BIon" in [18]) with contribution only from the gauge sector:

$$
\phi(r)=\int_{r}^{\infty} \frac{(p-2) c_{p}}{\sqrt{(p-2)^{2} c_{p}^{2}+r^{2(p-1)}}}, \quad y(r)=0 .
$$

This integral converges everywhere and has the form illustrated in figure 1 (for $p=3$ ). If it had been the scalar on the world volume which had the form illustrated in figure 2, such a solution appears to describe a configuration similar to the one illustrated in figure 11.a. It turns out that such a solution can be constructed by boosting the field configuration in the $\phi-y$ space along the lines explained in [18]. With some algebra, it is possible to summarize the set of solutions discussed in [18] as family of solutions parameterized by single variable $a$ :

$$
\phi(r)=\int_{r}^{\infty} d r \frac{1}{\sqrt{1-a+\left(\frac{r^{p-1}}{(p-2) c_{p}}\right)^{2}}}, \quad y(r)=\int_{r}^{\infty} d r \sqrt{\frac{a}{1-a+\left(\frac{r^{p-1}}{(p-2) c_{p}}\right)^{2}}}
$$


It is straightforward to verify that these solutions satisfy the equations of motion (11) and (2). These solutions have also been arranged so that the electric charge which couples to $\phi$ at the origin is precisely $c_{p}$. For the range of parameters $0<a<1$, the scalar field $y(r)$ essentially takes the form of figure 2. At $r=0$, the brane has a cusp, but takes on a finite value there. This provides a natural point for a string to end. Two sets of 3-branes in such a configuration admits a natural continuation with strings stretching between them, giving rise to a concrete realization of a configuration illustrated in figure 1.a. As a approaches 1, $y(r=0)$ grows indefinitely until it reaches the point $a=1$ at which we recover the BPS configuration $y=c_{p} / r^{p-1}$. For $a>1$, these solutions corresponds to the cantenoids (solutions of type illustrated in figure 1.c [17, 18]). In this paper, we will focus on the region $0 \leq a \leq 1$.

The configuration of branes for range of parameters $0 \leq a \leq 1$ appears to naturally capture the geometry illustrated in figure 1. 1. Even before doing any further analysis, however, we know that this can't be the proper description of the bending of the D-brane due to the tension of strings. This is because the lowest energy configuration of strings stretching between the branes is a BPS configuration, whereas the solution (3) is BPS only for $a=1$. It is nonetheless quite amusing and instructive to examine the static energies for these configurations and to understand precisely how this solution fails to describe the bending of D3-branes.

First, let us take a case where there is only one D3-brane and consider a string which ends on this D3-brane on one end and the other end extending infinitely. If the D3-brane takes on a shape described given by the solution (3), the total energy of the system will consist of energy density stored on the world volume of the D3-brane and the energy stored in the string which stretches to infinity

$$
E_{t o t}=\int d^{p} r T_{00}(r)+T \int_{y(0)}^{\infty} d y
$$

This expression diverges due to the contribution from the string extending indefinitely. A convenient way to subtract this infinite additive constant in energy is to subtract the energy of semi-infinitely extended string

$$
E=E_{t o t}-T \int_{0}^{\infty} d y=\int d^{p} r T_{00}(r)-T \int_{0}^{y(0)} d y=\int_{0}^{\infty} d r\left[r^{p-1} \Omega_{p-1} T_{00}(r)\right]-T y(0)
$$

The energy density of this system is given by

$$
T_{00}=T_{p}\left(\frac{1+(\nabla y)^{2}}{\sqrt{1+|\nabla y|^{2}-|\nabla \phi|^{2}+(\nabla y \cdot \nabla \phi)^{2}-(\nabla y)^{2}(\nabla \phi)^{2}}}-1\right) .
$$

For the configuration (3), the contribution for the energy density from the world volume of the brane is given by 


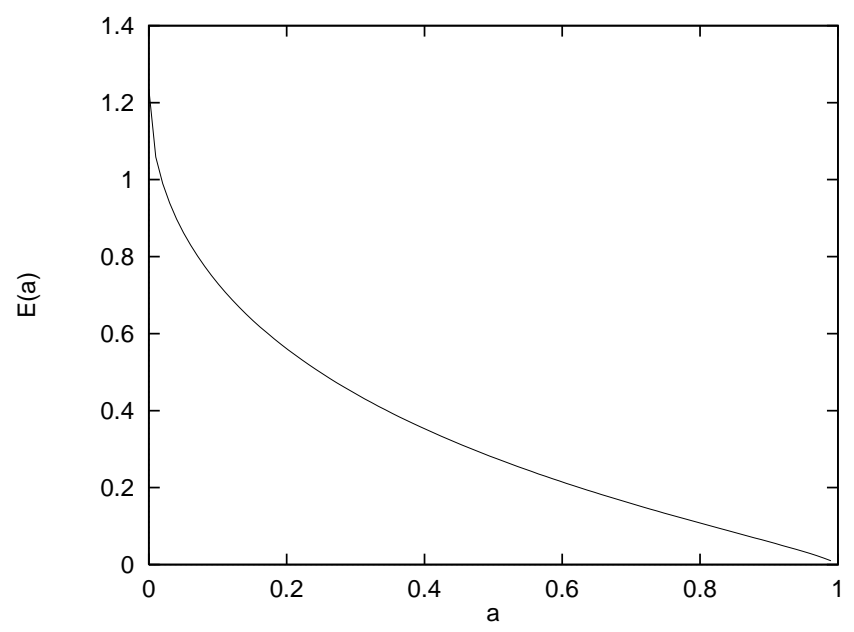

Figure 3: Combined energy in units of $T \sqrt{c_{3}}$ of bent D3-brane and stretched strings for one parameter family of configuration (3) as a function of the parameter $a$.

$$
\begin{aligned}
& r^{p-1} \Omega_{p-1} T_{00}(r) \\
& =\Omega_{p-1} T_{p}(p-2) c_{p}\left(-\left(\frac{r^{p-1}}{(p-2) c_{p}}\right)+\frac{a}{\sqrt{1-a+\left(\frac{r^{p-1}}{(p-2) c_{p}}\right)^{2}}}+\sqrt{1-a+\left(\frac{r^{p-1}}{(p-2) c_{p}}\right)^{2}}\right) \\
& =T\left(-\left(\frac{r^{p-1}}{(p-2) c_{p}}\right)+\frac{a}{\left.\sqrt{1-a+\left(\frac{r^{p-1}}{(p-2) c_{p}}\right)^{2}}+\sqrt{1-a+\left(\frac{r^{p-1}}{(p-2) c_{p}}\right)^{2}}\right)}\right.
\end{aligned}
$$

where we have used the fact that $\Omega_{p-1} T_{p}(p-2) c_{p}=T$. This expression can be integrated explicitly. Including the $T y(0)$ contribution and setting $p=3$, we find

$$
E(a)=\left(T \sqrt{c_{3}}\right) \frac{\Gamma\left(\frac{1}{4}\right)^{2}}{12 \sqrt{\pi}}(\sqrt{a}-2)(\sqrt{a}-1)(1-a)^{-1 / 4} .
$$

The form of this $E(a)$ is illustrated in figure 3, from which one can clearly observe that the energy is minimized at $a=1$. This is the point where the spike have been pulled all the way out to infinity and the brane configuration collapses to the simple expression $y=c_{p} / r^{p-2}$. This is to be expected since $a=1$ is the only BPS solution among the family of configurations parameterized by $a$.

The physical picture behind this phenomenon is simply that when a string attaches itself to a D-brane, the pull of the string tension will create a spike on the world volume of the D-brane. However, for finite $g$, the tension of the string is strong enough to pull the spike out of the D-brane indefinitely. If the original strings extends infinitely in the direction transverse to the brane, then the spike will grow infinitely in the direction of the string until it assumes the minimum energy configuration which is simply $y=c_{p} / r^{p-2}$. However, since $c_{p}$ is linear in $g$, the spike becomes narrower in the weak coupling limit. 
This picture leads to a slightly different interpretation of the spike than the authors of [17, 18], where because of the matching of the tension, the spike was interpreted as the string itself. Here, the spike is to be interpreted as a part of the D3-brane. That the tension matches exactly with the tension of fundamental strings is simply the reflection of the fact that D3brane must bend in such a way to generate precisely the tension exerted by the attached string in order to achieve static equilibrium. However, the distinction may not be so sharp when the radius of the spike becomes small. At length scale much smaller than the radius of the spike, one can physically identify the spike as being made of D3-brane by measuring its local Ramond-Ramond charge. At length scales much larger than the radius of the spike, however, the net Ramond-Ramond charge is cancelled by the brane on the other side of the tube. On the other hand, the tube will appear to carry Neveu-Schwarz charge through coupling with the world volume gauge field background. So from the consideration of charges, the spike, at length scales much larger than its radius, looks more like a string. Perhaps there is no sense in which the D3-brane spike can be distinguished from a fundamental string when the radius of the spike is smaller than the string scale.

With the information at hand, one can qualitatively address the situation where a string is stretching between pair of D3-branes separated by finite distance. The tension of the string will create a spike on D3-branes at both ends of the string. The tension of the string is strong enough to pull the D3-branes together until the length of the string shrinks to zero. Thus, one expects a geometry like the one illustrated in figure 1. b to be the final configuration. One might suppose that this configuration corresponds to pair of configuration of the form (3) for a particular value of $0<a<1$ so that the height of the spike is precisely half the distance between the D3-branes. The energy of this configuration can be computed by combining the integrated stress energy tensor from both the branes. Energies $E(a)$ illustrated in figure 3 can be interpreted here as the amount of excess energy with respect to the BPS energy of the brane configuration at the particular value of $a$. The fact that $E(a)$ is strictly positive for $0<a<1$ implies that this is not quite the equilibrium configuration for the brane. After the spike meets, the brane must adjust its shape slightly in order to achieve the lowest energy configuration. In the following sections, we will attempt to calculate the precise shape to which the branes relax in achieving such a state.

\section{A view from M-theory}

The main problem with the approach of the previous section was the fact that we were working with abelian Born-Infeld action although the system is best described by a nonabelian theory. In this section, we will consider a closely related problem of understanding the geometry where a string stretches between a pair of type IIA D2-branes. For such a system, one can bypass the need to use the non-abelian Born-Infeld action by going to the M-theory limit of the theory, where both the string and the D2-brane becomes an M-theory membrane. BPS configuration for these membranes can be realized explicitly as holomorphic 
embeddings of the world volume of these branes into spacetime [13].

Consider a configuration in type IIA theory where a string stretches between a pair of D2-brane which extends infinitely in $X_{1}$ and $X_{2}$ direction and is separated by some finite distance in $X_{3}$ direction. In the M-theory limit, the string becomes a "tube" of membrane which wraps around $X_{10}$ direction and extends in $X_{3}$ direction. The entire system of D2branes and string can be thought of as a single membrane object. In order to describe a BPS configuration of this membrane, it is convenient to introduce complex coordinates

$$
z=X_{1}+i X_{2}, \quad w=X_{3}+i X_{10} .
$$

Configuration of the type

$$
f(z, w)=0
$$

where $f(z, w)$ is a holomorphic function will correspond to a BPS configuration of a membrane in M-theory.

Since $X_{10}$ is a periodic variable, it is convenient to work instead with

$$
t=e^{w / R}
$$

where $R$ is the radius of the 11-th direction. Our goal then is to find a holomorphic function $f(z, t)$ which vanishes along the locus of the membrane world volume which arise from lifting the configuration of a string stretched between two D2-branes to M-theory. Finding $f(z, t)$ satisfying this requirement is analogous to the procedure for finding a five brane configuration which was described in [12], and we will use the same methods here. In particular, since there are two branches of membranes extending infinitely in the complex $z$-plane, we expect $f(z, t)$ to have two zeros for fixed value of $z$. Similarly, the membrane wraps the 11-th direction precisely once. Therefore, for fixed value of $t$, we expect $f(z, t)$ to have exactly one zero. Therefore, $f(z, t)$ must be at most quadratic in $t$ and linear in $z$. The most general holomorphic curve satisfying this requirement is

$$
f(z, t)=(a z+b) t^{2}+(c z+d) t+(e z+f) .
$$

At the zero of $e z+f, f(z, t)$ has a zero at $t=0$. In terms of $w=X_{3}+i X_{10}$ coordinate, this corresponds to $X_{3}=-\infty$ and implies the presence of a string stretching indefinitely in the $X_{3} \rightarrow-\infty$ direction. Similarly, at the zero of $a z+b$, the zero of $f(z, t)$ runs off to infinity. This implies the presence of a string stretching indefinitely to the $X_{3}=+\infty$ direction. Since we want the strings to stretch only between the two "would be" D2-branes, both of these features are undesirable. They can be eliminated by setting $a$ and $e$ to zero. Also, since the overall scale of $f(z, t)$ is irrelevant, we can set one of the remaining coefficients, say $f$ to 1 . The $b$ can be absorbed into the scale of of $t$. Similarly, coefficients $c$ and $d$ can be absorbed into the choice of scale and the origin of $z$. The holomorphic embedding of a membrane in $(z, t)$ plane which reduces to a string stretching between D2-branes in type IIA theory is therefore given by a curve

$$
t^{2}+2 z t+1=0,
$$




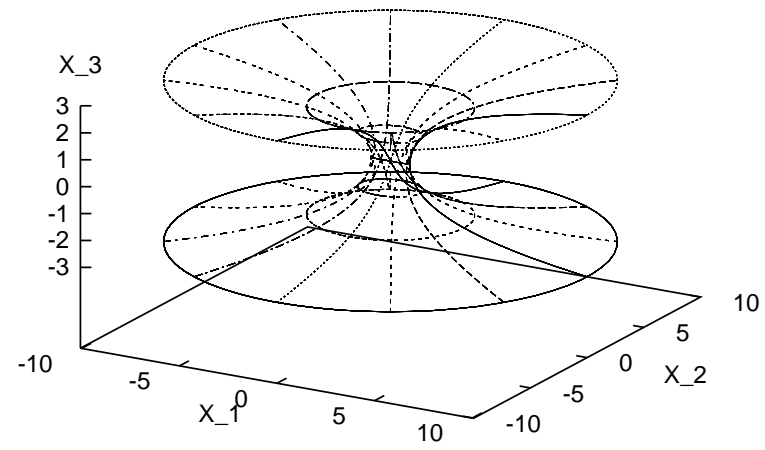

Figure 4: Projection to $\left(X_{1}, X_{2}, X_{3}\right)$ space for the holomorphic curve for the M-theory description of a string stretching between a pair of D2-branes

or equivalently,

$$
z=\frac{1}{2}\left(t+\frac{1}{t}\right)
$$

In terms of the original coordinates, this becomes

$$
\begin{aligned}
& X_{1}=\cosh \left(X_{3} / R\right) \cos \left(X_{10} / R\right) \\
& X_{2}=\sinh \left(X_{3} / R\right) \sin \left(X_{10} / R\right)
\end{aligned}
$$

This curve does not preserve rotational invariance in the $\left(X_{1}, X_{2}\right)$ plane. This might have been expected since we also broke the rotational invariance of $\left(X_{3}, X_{10}\right)$ plane by compactifying along the $X_{10}$ direction, and the holomorphic curve relates the two planes. In the weak coupling limit where we expect to recover a rotationally invariant string configuration, this asymmetry also disappears.

We illustrate the projection of this curve in the $\left(X_{1}, X_{2}, X_{3}\right)$ space in figure 4 . It has a feature that as $r=\left|X_{1}+i X_{2}\right|$ is taken to infinity, the value of $X_{3}$ grows like $\ln (r)$. This means that there is no clear notion of the "position of the branes at infinity," which will require additional care when taking the limit back to the weakly coupled type IIA description.

It is also instructive to examine the cross section of this curve with respect to $X_{1}=0$ plane or $X_{2}=0$ plane. For $X_{2}=0$, this curve reduces to

$$
X_{1}= \pm \cosh \left(X_{3} / R\right)
$$

and appears to describe a geometry similar to that of a cantenoidal configuration illustrated in figure 11.c. On the other hand, the cross section with $X_{1}=0$ plane takes the form

$$
X_{2}= \pm \sinh \left(X_{3} / R\right)
$$

Such a curve appears to cross each other at $X_{3}=0$. In the full M-theory description, however, these branches do not really cross, since they correspond to different points in the 
$X_{10}$ direction. In the limit where weakly coupled type IIA theory is recovered, however, the $X_{10}$ direction is shrinking and we expect crossing at $X_{3}=0$ to degenerate to a pinched brane configuration, similar to the ones discussed in the previous section. It should be also noted that when this geometry is projected onto $\left(X_{1}, X_{2}, X_{3}\right)$ space, this apparent crossing is required to undo the orientation reversal of the branes that would otherwise lead to a brane anti-brane configuration (figure 11.c).

Let us now imagine taking the limit of shrinking the radius of the 11-th dimension to zero size where we recover the weakly coupled type IIA description. The naive $R \rightarrow 0$ limit of (四) appears to collapse the locus of the membrane to $X_{3}=0$ plane. This problem is related to the fact that due to the logarithmic divergence of the brane coordinates, there is no well defined notion of the distance between the two branches of the curve at asymptotic region $r \gg 1$. In M-theory, choosing the distance between the brane is analogous to the choice of scale in asymptotically free theories, and one must perform a certain renormalization procedure in order to recover the weakly coupled type IIA description of D2-branes separated by a finite distance. One way to achieve this is to require that the brane passes through the point $\left(X_{1}, X_{2}, X_{3}\right)=(\bar{r}, 0, b)$ as we vary $R$. This amounts to rescaling (必) by a factor of $\bar{r} / \cosh (b / R)$ :

$$
X_{1}=\frac{\bar{r} \cosh \left(X_{3} / R\right) \cos \left(X_{10} / R\right)}{\cosh (b / R)}, \quad X_{2}=\frac{\bar{r} \sinh \left(X_{3} / R\right) \sin \left(X_{10} / R\right)}{\cosh (b / R)}
$$

With this chose of scales, $X_{1}$ and $X_{2}$ goes to in the $R \rightarrow 0$ limit for range of $X_{3}$ between $-b$ and $b$. This is the string stretching between two D2-branes. For $X_{3}$ slightly greater than $b$, both $X_{1}$ and $X_{2}$ diverges. This indicates the presence of D2-brane at $X_{3}= \pm b$.

It is also interesting to examine the asymptotic expansion of the curve (5) in the small $R$ limit. Such an expansion captures the information accessible from the weak coupling analysis. The $X_{2}=0$ cross section of the curve (5) is then given by

$$
X_{3}=R \cosh ^{-1}\left(X_{1} \cosh (b / R) / \bar{r}\right)
$$

which admits an asymptotic expansion

$$
X_{3}=b+R \ln (\bar{r} / r)+R\left(1-\frac{\bar{r}^{2}}{r^{2}}\right) e^{-2 b / R}+R\left(\frac{1}{2}-\frac{4 \bar{r}^{2}}{r^{2}}+\frac{3 \bar{r}^{4}}{2 r^{4}}\right) e^{-4 b / R}+\ldots
$$

Similarly, the $X_{1}=0$ cross section

$$
X_{3}=R \sinh ^{-1}\left(X_{1} \cosh (b / R) / \bar{r}\right)
$$

admits an asymptotic expansion

$$
X_{3}=b+R \ln (\bar{r} / r)+R\left(1+\frac{\bar{r}^{2}}{r^{2}}\right) e^{-2 b / R}+R\left(\frac{1}{2}+\frac{4 \bar{r}^{2}}{r^{2}}+\frac{3 \bar{r}^{4}}{2 r^{4}}\right) e^{-4 b / R}+\ldots
$$

In the $R \rightarrow 0$ limit, these expressions collapses to $X_{3}=b$ as it should. It is also interesting to note that a perturbative analysis would have only revealed the presence of the constant term 
and the logarithmic term. This is exactly the BPS configuration of the form $y=c_{p} / r^{p-2}$ described in [17, 18 for $p=2$. At this order, the geometry is rotationally invariant in the $\left(X_{1}, X_{2}\right)$-plane. All the other correction appears to be suppressed by factors of the form

$e^{-b / R}$. Such terms would have only been visible through non-perturbative analysis of the brane world volume field theory. It is also interesting to note that at the non-perturbative level, rotational symmetry in $\left(X_{1}, X_{2}\right)$-plane is broken. This is simply the manifestation of asymmetry between $\cosh \left(X_{3} / R\right)$ and $\sinh \left(X_{3} / r\right)$ cross sections for the M-theory membrane configuration (5).

\section{Brane configurations in type IIB theory}

The method of generating intersecting brane configuration as a limit of M-theory membrane configuration is remarkably powerful, where by taking advantage of holomorphicity, one is able to derive the full non-perturbative structure of the brane world volume field configuration. Unfortunately, this technique is limited in its applicability to class of configurations which admits a description in terms of either single membrane or a five-brane. In particular, it is not clear if this technique can be applied to study the configuration of our interest, namely string stretched between a pair of D3-branes in the type IIB theory. Also, in brane configuration where a fundamental string stretches between a pair of D-branes, the geometry in the weak coupling limit is flat as we saw in the case of D2-branes in the previous section. This is to be expected since the tension of D-branes grows like $1 / g$ in the weak coupling limit, whereas the fundamental string tension is invariant under the change in the strength of the coupling. In type IIB theory, on the other hand, one can keep the non-trivial geometry of branes intact even in the weak coupling limit by stretching a D-string instead of a fundamental string, since the tension of the D-string will also scale like $1 / g$ in that limit. In this section, we will examine this type of configurations in type IIB theory.

Ideally, one would like to study this problem using the full non-abelian Born-Infeld action. Presently, we are unable to carry out this program due to our inability to write down the non-abelian Born-Infeld action explicitly [22, 23]. However, for BPS states, the solution to the Maxwellian truncation of the abelian Born-Infeld theory is also the solution of the full Born-Infeld theory as was shown in [17, 18]. It is reasonable to expect similar relation to hold between non-abelian Born-Infeld theory and its Yang-Mills truncation (See Appendix A). Under this assumption, we will infer the geometry of the D3-branes from the BPS field configuration of Yang-Mills theories. Once these field configuration for Yang-Mills theories are identified, we will be able to compute their energies and verify the interpretation that they are being pulled by strings of appropriate tensions.

Let us begin by considering the situation where a D-string stretches between a pair of D3-branes, separated by a distance $2 b$. On the D3-brane world volume, this configuration is described by a magnetic monopole solution for $U(2)$ gauge theory coupled to adjoint Higgs field. The eigenvalues of the adjoint Higgs field at infinity will correspond to the distance 
separating the D3-brane at infinity.

Our task of determining the bent geometry of D3-branes is simplified dramatically in light of the fact that the field configuration of a magnetic monopole in $U(2)$ Yang-Mills theory is known explicitly in the form of the Prasad-Sommerfeld solution [24]. We can simply read off the D3-brane geometry from the field configuration of the Higgs scalar in the solution of 24.

The magnetic monopole of Prasad and Sommerfeld is a classical solution of the theory defined by an action

$$
S=-\frac{1}{e^{2}} \int d^{4} x \frac{1}{4}\left(F_{\mu \nu}^{a}\right)^{2}+\frac{1}{2}\left(D^{\mu} \varphi^{a}\right)^{2}
$$

where

$$
F_{\mu \nu}^{a}=\partial_{\mu} A_{\nu}^{a}-\partial_{\nu} A_{\mu}^{a}+\epsilon^{a b c} A_{\mu}^{b} A_{\nu}^{c}, \quad D_{\mu} \varphi^{a}=\partial_{\mu} \varphi^{a}+\epsilon^{a b c} A_{\mu}^{b} \varphi^{c}
$$

The solution is given in terms on an ansatz

$$
\begin{aligned}
A_{i}^{a} & =\epsilon_{a i j} \hat{r}_{j}[1-K(r)] / r \\
A_{0}^{a} & =\hat{r}_{a} J(r) / r \\
\varphi^{a} & =\hat{r}_{a} H(r) / r
\end{aligned}
$$

with $K(r), J(r)$, and $H(r)$ for the magnetic monopole given by the solution

$$
\begin{aligned}
K(r) & =C r / \sinh (C r) \\
J(r) & =0 \\
H(r) & =C r \operatorname{coth}(C r)-1
\end{aligned}
$$

where $C$ is a constant. In order to interpret this field configuration as a solution to the D-brane world volume theory, we need a precise dictionary of field variables. In order to find such a dictionary, consider expanding the abelian Born-Infeld action for the D3-brane

$$
\begin{aligned}
S & =T_{3} \sqrt{-\operatorname{det}\left(\eta_{\mu \nu}+T^{-1} F_{\mu \nu}+\partial_{\mu} X \partial_{\nu} X\right)} \approx T_{3}\left[\frac{1}{4} T^{-2} F^{2}+\frac{1}{2}(\partial X)^{2}\right] \\
& =T_{3} T^{-2}\left(\frac{1}{4} F^{2}+\frac{1}{2} T^{2}(\partial X)^{2}\right)
\end{aligned}
$$

Therefore, the appropriate identification is to take

$$
\varphi(r)=T X(r), \quad \frac{1}{e^{2}}=T_{3} T^{-2}
$$

The adjoint Higgs scalar on the D3-brane world volume therefore takes the form

$$
X(r)=T^{-1} \tau^{a} \hat{r}^{a} H(r) / r=\tau^{a} \hat{r}^{a} T^{-1}\left(C \operatorname{coth}(C r)-\frac{1}{r}\right)
$$

where $\tau^{a}$ are the generators for the $S U(2)$ Lie algebra.

The form of $X(r)$ found above contains components in all directions in the space of $S U(2)$ Lie algebra. One would like to diagonalize this field so that the eigenvalues can be interpreted 


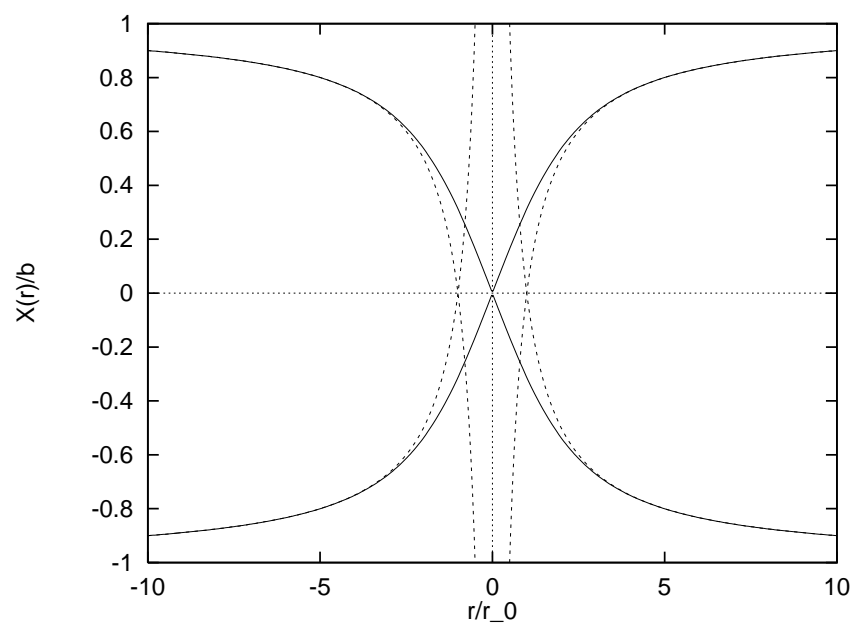

Figure 5: Deformation of D3-brane by the tension of a string stretching between them. The solid line is the shape inferred from the field configuration of the Higgs scalar in the Prasad Sommerfeld solution. The dotted line is the BPS configuration $X=c_{p} / r^{p-2}$ for the abelian Born-Infeld theory. The scale factor $r_{0}$ is $g / T b$ for the fundamental string and $1 / T b$ for the D-string.

as the position of the D3-brane. Such a diagonalization can be carried out on an arbitrary coordinate patch by performing appropriate gauge transformation?. At least locally, we find that the embedding of D3-brane world volume into transverse space is specified according to

$$
X(r)= \pm T^{-1}\left(C \operatorname{coth}(C r)-\frac{1}{r}\right) .
$$

As $r$ is taken to infinity, $X(r)$ approaches $T^{-1} C$. We can therefore set the value of $C$ in terms of distance $\Delta X=2 b$ between the D3-branes as $C=T \Delta X / 2$. The leading asymptotic dependence of $X(r)$ on $r$ goes as $T^{-1} / r$. Except for the factor of $g$ which accounts for the difference between the fundamental string and a D-string, this is precisely equivalent to the field configuration $X(r)=c_{p} / r^{p-2}$ encountered earlier in the context of BPS configurations in abelian Born-Infeld theories.

Near $r=0, X(r)$ behaves like

$$
X(r) \approx \frac{1}{12} T(\Delta X)^{2} r
$$

The expectation therefore is for radius of the D3-brane spike to shrink linearly to zero as one approaches the point halfway between the two branes. The form of $X(r)$ is illustrated in figure 5 .

Given the exact field configuration, one can immediately calculate its energy. The canonical Hamiltonian energy of (6) for the ansatz (7) is given by

$$
E=\frac{1}{e^{2}} \int d^{3} r\left(K^{\prime 2}+\frac{\left(K^{2}-1\right)^{2}}{2 r^{2}}-\frac{J^{2} K^{2}}{r^{2}}-\frac{\left(r J^{\prime}-J\right)^{2}}{2 r^{2}}+\frac{H^{2} K^{2}}{r^{2}}+\frac{\left(r H^{\prime}-H\right)^{2}}{2 r^{2}}\right)
$$

\footnotetext{
${ }^{3}$ In attempting to perform such a gauge transformation globally, one encounters a Dirac-string singularity characteristic of magnetic charges. This can be thought of heuristically as the continuation of the D-string.
} 
For the monopole solution (8), this is simply

$$
E=\frac{4 \pi C}{e^{2}}=4 \pi\left(T_{3} T^{-2}\right)(T \Delta X / 2)=\frac{T}{g} \Delta X .
$$

This is precisely the energy of D-string stretching for the length $\Delta X$ ! The fact that this energy worked out to an expected value suggests that we have found the correct configuration of D3-branes sustaining precisely the amount of stress needed to balance the tension of the D-string attached to its world volume.

Let us now discuss the situation where a fundamental string stretches between a pair of D3-branes. The most efficient method for studying this situation is to act on (9) by electric-magnetic duality transformation. Then, after appropriate field redefinitions, the same solution (8) can be used to describe the configuration of interest.

Under electric-magnetic duality, the action (9) transforms according to rules 26]

$$
\begin{aligned}
& T_{3}=\frac{1}{2 \pi g} T^{2} \rightarrow \frac{1}{2 \pi} T^{2} g \\
& \sqrt{-\operatorname{det}\left(\eta_{\mu \nu}+\partial_{\mu} X \partial_{\nu} X+T^{-1} F_{\mu \nu}\right)} \rightarrow \sqrt{-\operatorname{det}\left(g^{-1} \eta_{\mu \nu}+g^{-1} \partial_{\mu} X \partial_{\nu} X+T^{-1} F_{\mu \nu}\right)} \\
& \\
& \approx T^{-2}\left(\frac{1}{4} F^{2}+\frac{1}{2} T^{2} g^{-2}(\partial X)^{2}\right) .
\end{aligned}
$$

So the dual action becomes

$$
S=\frac{1}{2 \pi} g\left(\frac{1}{4} F^{2}+\frac{1}{2} T^{2} g^{-2}(\partial X)^{2}\right)
$$

with the identification

$$
\varphi(r)=T g^{-1} X(r), \quad C=T g^{-1} \Delta X / 2, \quad \frac{1}{e^{2}}=\frac{1}{2 \pi} g .
$$

This time, the energy is

$$
E=\frac{4 \pi C}{e^{2}}=T \Delta X
$$

which is precisely the energy of fundamental string stretching for a length $\Delta X$.

It is interesting to compare the shape of the deformed D3-brane due to D-string and the fundamental string. For the D-string we found

$$
X_{D}(r)=b \operatorname{coth}(T b r)-T^{-1} \frac{1}{r}
$$

whereas for the fundamental string, we found

$$
X_{F}(r)=b \operatorname{coth}(T b r / g)-g T^{-1} \frac{1}{r}
$$

where $b$ is the position of D3-brane at infinity. The main difference between the two field configurations is its dependence on the coupling. For the D1-string, the shape of D3-brane 
is independent of $g$. Therefore, the bending will persist in even in the $g \rightarrow 0$ limit. For the fundamental string, on the other hand, $X_{F}(r) \rightarrow b$ as $g \rightarrow 0$. the asymptotic expansion near $g=0$ is

$$
X_{F}(r)=b+g T^{-1} \frac{1}{r}+2 b e^{-2 T b r / g}+2 b e^{4 T b r / g}+\ldots .
$$

Just as in the case of D2-brane in type IIA theory, we find that only the first two terms in this series is perturbative, and to this level of approximation, the field configuration is identical to the form $c_{p} / r^{p-2}$ discussed earlier. The problem that such a configuration was unsuitable for describing strings stretching between pair of D3-brane appears to be resolved entirely through non-perturbative effects.

Finally, let us comment on the issue of whether brane configuration (10) and (11) is physically distinguishable from the configuration $c_{p} / r^{p-2}$ of [17, 18] in light of the fact that our ability to probe short distances is limited by the length-scales of the probe itself. To facilitate the comparison, we also included the plot of $c_{p} / r^{p-2}$ in figure 5 . At the point where the two branches meet, configurations (10) and (11) differs from $c_{p} / r^{p-2}$ by a quantity of order $r_{0}$. Since $r_{0}$ is different for the fundamental string and the D-string, let us examine each cases separately.

For the case of fundamental string stretching between the D3-branes, $r_{0}=g / T b$. In the weak coupling limit, this is smaller than the characteristic thickness of the fundamental string itself. The difference between (11) and $c_{p} / r^{p-2}$ is buried within the stringy/brany "fuzz" and appears not to have observable consequences.

In the case of D-string stretching between the D3-branes, on the other hand, $r_{0}=1 / \mathrm{Tb}$ is independent of $g$. Although $r_{0}$ is of order string scale and appears to hide beneath the stringy halo, it is much larger in comparison to the length scales accessible by say a D-string

probe which goes as $\sqrt{g / T}$ [27] in the weak coupling limit. We therefore conclude that the feature of (10) near $r=0$ is real and observable, at least until the radius $r$ shrinks to the characteristic thickness of the D-string $\sqrt{g / T}$.

\section{Discussion}

In this article, we examined the effect of bending of branes when a string attaches one of its endpoints and pulls by its own tension. For a system consisting of a single D-brane being pulled by a string which extends semi-infinitely, one can analyze its static equilibrium using abelian Born-Infeld action, as was done in [17, 18]. The picture which emerges from such an analysis is that the string tension is strong enough to pull the brane world volume all the way to infinity, provided the string coupling constant is non-zero.

The picture needs to be modified somewhat in order to discuss the geometry of a string stretching between a pair of D-branes, causing the branes to bend. In order to analyze this system along the lines of [17, 18], full non-abelian generalization of the Born-Infeld action is required. Currently, we are unable to carry out such a program due to our inability to write down such an action (See Appendix A). In this article, we side-stepped this problem 
by 1) considering a system of parallel D2 brane supporting a stretched string which admits a full non-perturbative treatment in the M-theory limit, and 2) considering the Yang-Mills truncation of the "would be" Born-Infeld action and assuming that for BPS configurations, Yang-Mills theory will give the correct answer. Here, we took advantage of the known explicit forms of $\mathrm{SU}(2)$ magnetic monopole field configuration. In particular, we were able to read off the embeddings of the D3-brane world volume into spacetime directly from the field configuration of the adjoint scalar field of the magnetic monopole. Both of these approaches have allowed us to construct explicit expressions describing the shape of the D2-branes and D3-branes in type IIA and IIB theories, respectively. In the case of a fundamental string stretching between the branes, however, the effect of bending becomes arbitrary small in the weak coupling limit and the geometry is essentially trivial. For the type IIB theory, however, one can suspend a D-string instead of a fundamental string. The non-trivial bending persists to the weak coupling limit and is physically observable from scattering of brane probes.

The approach of inferring brane configurations form the field configurations of magnetic monopole solutions can be generalized to dyon solutions, multi-monopole solutions, and larger gauge groups. These should correspond to $(p, q)$ strings, multiple strings, and multiple D3-branes, respectively, in the D-brane language. One could also imagine stretching fundamental strings for D-branes other than the D3-branes. However, in the weak coupling limit, all non-trivial geometry is expected to be hidden inside a stringy halo.

Although we presented the M-theory technique for describing the type IIA D2-branes and the magnetic monopole technique for describing the type IIB D3-branes as independent methods, the two are clearly related, since one can map from one to the other by chain of dualities. These systems are also closely related to pair of orthogonal D2-branes intersecting at a point whose BPS deformation also admits simple holomorphic descriptions [17]. The main difficulty of applying the language of holomorphic curves to D3-branes is the fact that such curves are naturally even dimensional and one must perform at least one T-duality transformation which obscures the distinction between spatial and gauge configurations. Yet there is a sense in which algebraic curves appears in the construction of magnetic monopoles 28, 29, 30. It would be very interesting to understand the unifying structure which underlies these different approaches.

\section{Acknowledgments}

I would like to thank Per Berglund, John Brodie, Sean Carroll, Steve Giddings, David Gross, Hiroshi Ooguri, Gary Horowitz, Juan Maldacena, Rob Myers, Amanda Peet, Phillipe Pouliot, and Washington Taylor for comments and useful discussions. This work was supported in part by the NSF grant PHY94-07194. 


\section{Appendix A Monopole solutions of non-abelian Born- Infeld action}

Throughout this article, we referred to the non-abelian generalization of the Born-Infeld action as the necessary framework for studying the geometry of parallel branes suspending a string. In generalizing the Born-Infeld action to its non-abelian counterpart, one must provide additional information specifying the order in the product of non-commutative factors. Attempts have been made by many authors to resolve this ambiguity [31, 32, 33], but only terms up to order $F^{4}$ were properly understood.

Recently, Tseytlin has proposed an explicit form for the non-abelian Born-Infeld action 22]

$$
S=T_{p} \int d^{p+1} x \operatorname{STr} \sqrt{-\operatorname{det}\left(\eta_{\mu \nu}+T^{-1} F_{\mu \nu}\right)}
$$

where the determinant is over the Lorenz indices $(\mu, \nu)$ and "STr" indicates that the trace over gauge indices is to be taken after symmetrizing over all permutations of the non-commutative products. Although this action has passed a number of consistency checks, a discrepancy was noted in [23] for the fluctuation spectrum of this model around a background of constant magnetic flux. Some modification of (12) appears to be necessary in order to resolve this discrepancy in full.

Although it is not clear how (12) will be modified when the discrepancy of [23] is fully resolved, this action appears to already exhibit many desirable features. For example, abelian Born-Infeld action has the property that a BPS configuration of its Maxwellian truncation satisfies the equation of motion of the full Born-Infeld action. This led us to conjecture that a BPS solution of Yang-Mills action is also a BPS configuration of the non-abelian BornInfeld action. It turns out that this property holds for the non-abelian Born-Infeld action of Tseytlin (12), as we will demonstrate in this appendix. 7

The world volume theory on D3-brane contains 6 adjoint scalars. For our purpose, we can set all but one of these adjoint scalar to zero. A convenient way to write down the DBI action then is to think of it as a dimensional reduction of $4+1$ dimensional Born-Infeld action

$$
S=\operatorname{STr} \sqrt{-\operatorname{det}\left(\eta_{a b}+T^{-1} F_{a b}\right)}
$$

where $(a, b)$ goes from 0 to 4 and

$$
F_{a b}=\left(\begin{array}{ccccc}
0 & F_{01} & F_{02} & F_{03} & T D_{0} X \\
-F_{01} & 0 & F_{12} & F_{13} & T D_{1} X \\
-F_{02} & -F_{12} & 0 & F_{23} & T D_{2} X \\
-F_{03} & -F_{13} & -F_{23} & 0 & T D_{3} X \\
-T D_{0} X & -T D_{1} X & -T D_{2} X & -T D_{3} X & 0
\end{array}\right) .
$$

For the static magnetic monopole solution, we can set $F_{0 i}$ and $T D_{0} X$ to zero, and the action reduces to the determinant of lower right $4 \times 4$ block. Now, the magnetic monopole solution

\footnotetext{
${ }^{4}$ I am especially indebted to Rob Myers for extended discussions on the contents of this appendix.
} 
of Prasad and Sommerfeld satisfies the identity

$$
B_{i}= \pm \frac{1}{2} \epsilon^{i j k} F_{j k}= \pm T D_{i} X
$$

or equivalently

$$
F_{i j}= \pm \frac{1}{2} \epsilon_{i j k l} F^{k l} .
$$

This is the familiar BPS condition which follows from supersymmetry.

In deriving the equation of motion for (12), we follow the approach of [23] where we take the expression inside the symmetrized trace as if they are abelian until the end of the calculation, at which point we will assign a singe power of $\tau^{a}$ for each factor of $F$ and sum over traces of all permutations of the non-abelian factors. The variation of (12) with respect to the fields inside the symmetrized trace is simply

$$
\delta S=\operatorname{STr}\left[\sqrt{-\operatorname{det}\left(\eta_{a b}+T^{-1} F_{a b}^{0}\right)}\left(\eta+T^{-1} F^{0}\right)_{a b}^{-1}\left(D^{a} \delta A^{b}-D^{b} \delta A^{a}\right)\right] .
$$

Self-dual field configuration has a unique property that the determinant factor is a perfect square:

$$
-\operatorname{det}\left(\eta_{a b}+T^{-1} F_{a b}^{0}\right)=\left(1+\left(T^{-1} F_{12}^{0}\right)^{2}+\left(T^{-1} F_{13}^{0}\right)^{2}+\left(T^{-1} F_{23}^{0}\right)^{2}\right)^{2} .
$$

Combining this with the fact that

$$
\frac{1}{2}\left(\left(\eta+T^{-1} F^{0}\right)_{a b}^{-1}-(a \leftrightarrow b)\right)=\frac{T^{-1} F_{a b}}{1+\left(T^{-1} F_{12}^{0}\right)^{2}+\left(T^{-1} F_{13}^{0}\right)^{2}+\left(T^{-1} F_{23}^{0}\right)^{2}}
$$

allows us to simplify (13) to read

$$
\delta S=\mathrm{S} \operatorname{Tr}\left[T^{-2} F_{a b}^{0} D^{a} \delta A^{b}\right] .
$$

At this point, since we only have two factors of the non-commutative elements, there is no need to distinguish the symmetrized trace and the ordinary trace. Now one can apply ordinary integration by parts to obtain the equation of motion

$$
D^{a} F_{a b}^{0}=0
$$

which is precisely identical to the equation of motion for the Yang-Mills action. Since the solution of Prasad and Sommerfield satisfies the equation of motion of the Yang-Mills action exactly, and since it is a self-dual field configuration, it follows that it also satisfies the equation of motion of (12) exactly.

Although we focused on purely magnetic solutions, one can generalize this argument to the dyon solution where the electric field is also turned on [24]. Such a solution is best thought of as the boost of the magnetic monopole solution in the 04-plane. It is trivial to repeat the argument replacing $F_{a b}$ with $\left(\Lambda^{-1} F \Lambda\right)_{a b}$.

It would be extremely interesting to understand the resolution of discrepancy noted in [23, and how such a resolution affects the result of this appendix. 


\section{References}

[1] J. Polchinski, "Dirichlet-Branes and Ramond-Ramond Charges," Phys. Rev. Lett. 75 (1995) 4724.

[2] E. Witten, "Bound States of Strings and p-Branes," Nucl. Phys. B460 (1996) 335, hep-th/9510135.

[3] M. Bershadsky, V. Sadov and C. Vafa, "D-Strings on D-Manifolds," Nucl. Phys. B463 (1996) 398-414, hep-th/9510225.

[4] A. Hanany and E. Witten, "Type IIB Superstrings, BPS Monopoles, and ThreeDimensional Gauge Dynamics," Nucl. Phys. B492 (1997) 152-190, hep-th/9611230.

[5] S. Elitzur, A. Giveon, D. Kutasov, "Branes and N=1 duality in string theory," Phys. Lett. B400 (1997) 269-274, hep-th/9702014.

[6] A. Strominger, "Open p-Branes," Phys. Lett. B383 (1996) 44-47, hep-th/9512059.

[7] M. B. Green and M. Gutperle, "Comments on Three-Branes," Phys. Lett. B377 (1996) 28-35, hep-th/9602077.

[8] D-E. Diaconescu, "D-branes, Monopoles, and Nahm equations," hep-th/9608163.

[9] C. Bachas, M. Douglas, and M. Green, "Anomalous Creation of Branes," JHEP 07 (1997) 002, hep-th/9705074.

[10] U. Danielsson, G. Ferretti, and I. R. Klebanov, "Creation of Fundamental Strings by Crossing D-branes," Phys. Rev. Lett. 79 (1997) 1984-1987, hep-th/9705084.

[11] O. Bergman, M. Gaberdiel, and G. Lifschytz, "Branes, Orientifolds and the Creation of Elementary Strings," hep-th/9705130.

[12] E. Witten, "Solutions of Four-Dimensional Field Theories via M-Theory," Nucl. Phys. B500 (1997) 3-42, hep-th/9703166.

[13] K. Becker, M. Becker, and A. Strominger, "Five-Branes, Membranes and Nonperturbative String Theory," Nucl. Phys. B456 (1995) 130-152, hep-th/9507158.

[14] D.-E. Diaconescu and N. Seiberg, "The Coulomb Branch of $(4,4)$ Supersymmetric Field Theories in Two Dimensions," JHEP 07 (1997) 001, hep-th/9707158, J. H. Brodie, "Two-Dimensional Mirror Symmetry from M-Theory," hep-th/9709228; M. Alishahiha, "N=(4,4) 2-D Supersymetric Gauge Theory and Brane Configuration," hep-th/9710020.

[15] S. B. Giddings, "Scattering Ripples from Branes," Phys. Rev. D55 (1997) 6367-6373, hep-th/9612022. 
[16] S. Carroll and M. Trodden, "Dirichlet Topological Defects," NSF-ITP-97-117, hep-th/9711099.

[17] C. G. Callan and J. M. Maldacena, "Brane Dynamics from the Born-Infeld Action," hep-th/9708147.

[18] G. W. Gibbons, "Born-Infeld Particles and Dirichlet p-Branes," hep-th/9709027.

[19] P. S. Howe, N. D. Lambert, and P. C. West, "The Selfdual String Soliton, hep-th/9709014; "The Three-Brane Soliton of the M-Five-Brane, hep-th/9710033.

[20] S. Lee, A. Peet, and L. Thorlacius, "Brane Waves and Strings," hep-th/9710097.

[21] L. Thorlacius, "Born-Infeld String as a Boundary Conformal Field Theory," hep-th/9710181.

[22] A. A. Tseytlin, "On Non-Abelian Generalisation of Born-Infeld Action in String Theory," Nucl. Phys. B501 (1997) 41-52, hep-th/9701125.

[23] A. Hashimoto and W. Taylor, IV, "Fluctuation Spectra of Tilted and Intersecting Dbranes from the Born-Infeld Action," hep-th/9703217.

[24] M. K. Prasad and C. M. Sommerfield, "An Exact Classical Solution for the 't Hooft Monopole and the Julia-Zee Dyon," Phys. Rev. Lett. 35 (1975) 760-762.

[25] M. Born and L. Infeld, "Foundations of the New Field Theory," Proc. Roy. Soc. Lond. A144 (1934) 425-451.

[26] A.A. Tseytlin, "Selfduality of Born-Infeld Action and Dirichlet 3-Brane of Type IIB Superstring Theory," Nucl. Phys. B469 (1996) 51-67, hep-th/9602064.

[27] M. R. Douglas, D. Kabat, P. Pouliot, and S. Shenker, "D-Branes and Short Distances in String Theory," Nucl. Phys. B485 (1997) 85-127, hep-th/9608024.

[28] N. J. Hitchin, "On the Construction of Monopoles," Comm. Math. Phys. 89 (1983) 145-190.

[29] J. Hurtbuise, "The Classification of Monopoles for the Classical Groups," Comm. Math. Phys. 120 (1989) 613-641.

[30] S. K. Donaldson and P. B. Kronheimer, The Geometry of Four-Manifolds, Oxford University Press, 1990.

[31] E. S. Fradkin, and A. A. Tseytlin, Phys. Lett. B163 (1985) 123.

[32] A. A. Tseytlin, Nucl. Phys. B276 (1986) 391, Erratum-ibid B291 (1987) 876.

[33] P. C. Argyres and C. R. Nappi, Nucl. Phys. B330 (1990) 151. 